\title{
A NOTE ON RIEMANN INTEGRABILITY
}

\author{
G. A. BEER \\ Department of Mathematics \\ California State University \\ Los Angeles, California 90032
}

(Received November 21, 1977)

ABSTRACT. In this note we define Riemann integrability for real valued functions defined on a compact metric space accompanied by a finite Borel measure. If the measure of each open ball equals the measure of its corresponding closed ball, then a bounded function is Riemann integrable if and only if its set of points of discontinuity has measure zero.

Let $\mathcal{A}$ denote the algebra of sets generated by the open and closed subintervals of an interval $[a, b]$. A bounded real valued function $f$ defined on $[a, b]$ is Riemann integrable if for each positive $\varepsilon$, there exist two functions $\phi$ and $\psi$ that are linear combinations of characteristic functions of sets in $\mathcal{d}$ satisfying $\phi \leq f \leq \psi$ and

$$
\int_{a}^{b} \psi d m-\int_{a}^{b} \phi d m<\varepsilon
$$

where $m$ denotes ordinary Lebesgue measure. Riemann integrability may be defined in an analagous way for real valued functions defined on a compact metric space $K$ accompanied by a finite Borel measure. If we make a simple 
assumption about the balls of $K$, then the following famous theorem of Lebesgue extends: a bounded real valued function $f$ defined on $[a, b]$ is Riemann integrable if and only if the set of points at which $f$ is not continuous has Lebesgue measure zero.

Suppose that $K$ is a compact metric space and $\mu$ is a finite Borel measure on $K$. Let $B_{r}(x)=\{y: d(x, y)<r\}$ and $\bar{B}_{r}(x)=\{y: d(x, y) \leq r\}$ denote the open and closed balls of radius $r$ about a point $x$ in $K$. Let $\mathcal{\&}$ denote the algebra generated by all such balls. Any element of $\mathcal{E}$ is of the form

$$
1 \leq i \leq m \quad 1 \leq \hat{k} \leq n_{i} A_{i k}
$$

where $A_{i k}$ is a ball or its complement and $\left\{m, n_{1}, \ldots, n_{m}\right\}$ are positive integers. A step function is a linear combination of characteristic functions determined by elements of $\mathcal{L}$. Hence a step function $\phi$ has the form $\sum d_{i} x_{i}$ where each $d_{i}$ is real and $A_{i} \in \mathcal{E}$. Since $\mathcal{L}$ is an algebra, the $\left\{A_{i}\right\}$ can be taken to be pairwise disjoint. It is easy to see that if $\phi$ and $\psi$ are step functions, then so are $\phi+\psi, \phi-\psi$, inf $\{\phi, \psi\}$, and $\sup \{\phi, \psi\}$.

DEFINITION. A bounded real valued function $f$ defined on $K$ is Riemann integrable if for each positive $\varepsilon$ there exist step functions $\phi$ and $\psi$ such that $\phi \leq f \leq \psi$ and $\int \psi d \mu-\int \phi d \mu<\varepsilon$.

Given a bounded real valued function $f$ defined on $K$, the upper envelope $h$ of $f$ is the function defined by

$$
h(x)=\inf _{\delta>0} \sup _{y \in B_{\delta}(x)} f(y) \quad x \in K
$$


and the lower envelope $g$ of $f$ is defined by

$$
g(x)=\sup _{\delta>0} \inf _{y \in B_{\delta}(x)} f(y) \quad x \in K
$$

It is well known that $h$ is upper semicontinuous, $g$ is lower semicontinuous, $g(x) \leq f(x) \leq h(x)$ for each $x$, and $g(x)=h(x)$ if and only if $f$ is continuous at $x$ (see Royden [1, p.49]).

THEOREM. Suppose $\mu\left(B_{r}(x)\right)=\mu\left(\bar{B}_{r}(x)\right)$ for each $x$ in $K$ and for each positive $r$. A bounded real valued function $f$ defined on $K$ is Riemann integrable if and only if the set of points at which $f$ is discontinuous has $\mu$-measure zero.

Proof. Let $h$ be the upper envelope of $f$ and $g$ its lower envelope. Let $\psi$ be any step function that exceeds $f$. Since each member of \& can be expressed in the form depicted in (1), the condition on the balls of $\mathrm{K}$ implies that each member of $\mathcal{L}$ is the union of an open set and a set of $\mu$-measure zero. It follows that $\psi$ can be represented as

$$
\sum_{j=1}^{n} a_{j} x_{A_{j}}
$$

where (i) $A_{j}$ is an open set for $1 \leq j \leq m$ (ii) $\mu\left(A_{j}\right)=0$ for $m<j \leq n$ (iii) $\left\{A_{1}, A_{2}, \ldots, A_{n}\right\}$ partition $K$.

$$
\text { Let } x \in \bigcup_{j=1}^{m} A_{j} \text {. Since } \psi \text { is constant near } x \text {, there exists } \delta>0
$$

such that $\psi(x) \geq \sup _{y \in B_{\delta}(x)} f(y)$ so that $\psi(x) \geq h(x)$. Hence, $\mu\{x: \psi(x)<h(x)\}=0$, and we have $\int \psi d \mu \geq \int h d \mu$. We now construct a decreasing sequence of step functions converging pointwise to $h$ so that 
inf $\left\{\int \psi d \mu: \psi \geq f\right.$ and $\psi$ is a step function $\}=\int h d \mu$.

Let $\mathrm{N}$ be a fixed positive integer. Let $\left.{ } \mathrm{B}_{\mathrm{r}_{1}}\left(\mathrm{x}_{1}\right), \ldots, \mathrm{B}_{\mathrm{r}_{\mathrm{m}}}\left(\mathrm{x}_{\mathrm{m}}\right)\right\}$ be a cover of $K$ by balls of radius at most $1 / N$ such that if $y \in B_{r_{i}}\left(x_{i}\right)$, then $h(y)<h\left(x_{i}\right)+1 / N$. Now let $\theta_{N}: K \rightarrow R$ be the step function described by ${ }{ }_{N}(x)=\inf \left\{h\left(x_{i}\right)+1 / N: x \in B_{r_{i}}\left(x_{i}\right)\right\}$. Define $\psi_{N}$ to be ${ }_{\mathrm{N}} \cdot$ Given any positive integer $p$, define ${ }^{\theta}{ }_{\mathrm{N}+\mathrm{p}}$ as above, and let $\psi_{\mathrm{N}+\mathrm{p}}$ be inf $\left\{\theta_{\mathrm{N}+\mathrm{p}}, \psi_{\mathrm{N}+\mathrm{p}-1}\right\}$. Clearly, for each $\mathrm{p} \psi_{\mathrm{N}+\mathrm{p}}$ is a step function, and $\psi_{\mathrm{N}+\mathrm{p}} \geq \psi_{\mathrm{N}+\mathrm{p}+1} \geq \mathrm{h}$. To establish the pointwise convergence, suppose to the contrary that for some $x_{0}$ in $K$ and $\varepsilon>0$ we have for each $p$

$$
\psi_{N+p}\left(x_{0}\right)>h\left(x_{0}\right)+2 \varepsilon
$$

Pick $n$ so large that $1 / \mathrm{n}<\varepsilon$. There exists a point $x_{n}$ such that $d\left(x_{0}, x_{n}\right)<1 / n$ and $\psi_{n}\left(x_{0}\right) \leq h\left(x_{n}\right)+1 / n$. Clearly, $h\left(x_{n}\right)>h\left(x_{0}\right)+\varepsilon$ which violates the upper semicontinuity of $h$. Hence, $\left\{\psi_{n}\right\}$ is the desired sequence.

Using the above technique we can show in the same manner that $\int g \mathrm{~d} \mu=\sup \left\{\int \phi \mathrm{d} \mu: \phi \leq f\right.$ and $\phi$ is a step function $\}$. The proof is now completed by observing the equivalence of the following statements:

(i) $f$ is Riemann integrable (ii) $\int g d \mu=\int h d \mu$ (iii) $f$ is continuous except at a set of points of $\mu$-measure zero.

A simple example shows that the theorem need not hold if our condition on the balls of the metric space is omitted. Let $K$ be the closed unit disc in the plane with the usual metric. If $B$ is a Borel subset of $K$, define $\mu(B)$ to be $\mu_{1}\left(B \cap\left\{(x, y): x^{2}+y^{2}=1\right\}\right)+\mu_{2}\left\{B \cap\left\{(x, y): x^{2}+y^{2}\right.\right.$ 
< 1\} where $\mu_{2}$ is two dimensional Lebesgue measure and $\mu_{1}$ is one dimensional Lebesgue measure, considering the circle as having measure $2 \pi$. Then the characteristic function of the unit circle is Riemann integrable (being a step function), but its set of discontinuities has measure $2 \pi$.

\section{REFERENCES}

1. H. L. Royden. Real Analysis, Macmillan, New York, 1968.

KEY WORDS AND PHRASES. Riemann integrable functions on a compact metric space, Compact metric space with Borel measure.

AMS (MOS) SUBJECT CLASSIFICATIONS (1970). $28 A 25$. 


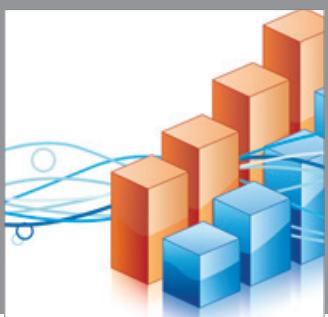

Advances in

Operations Research

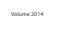

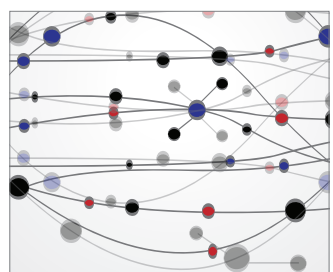

\section{The Scientific} World Journal
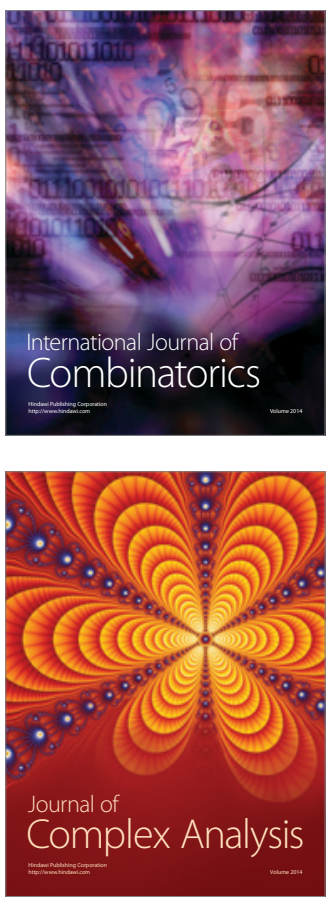

International Journal of

Mathematics and

Mathematical

Sciences
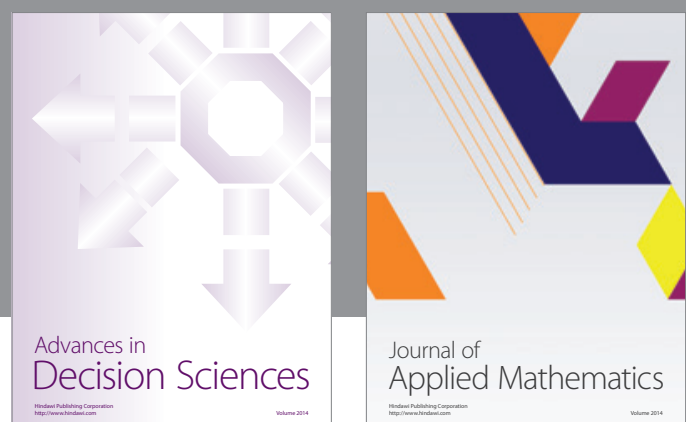

Journal of

Applied Mathematics
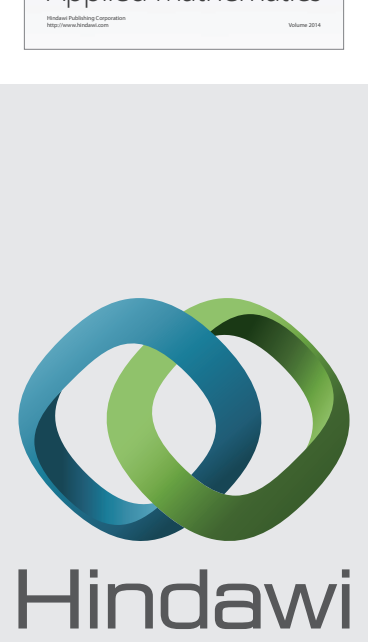

Submit your manuscripts at http://www.hindawi.com
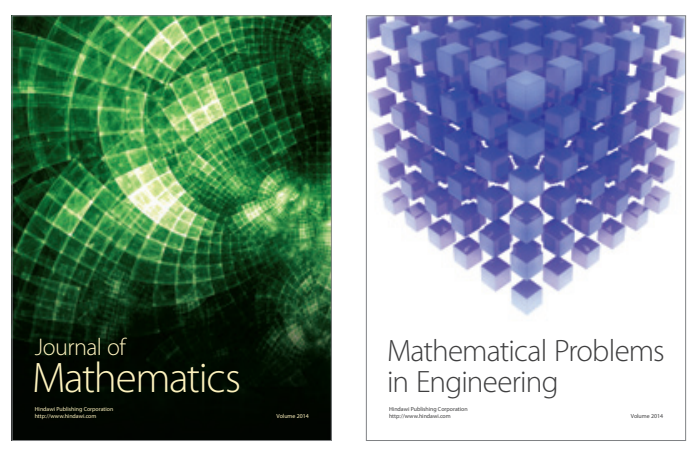

Mathematical Problems in Engineering
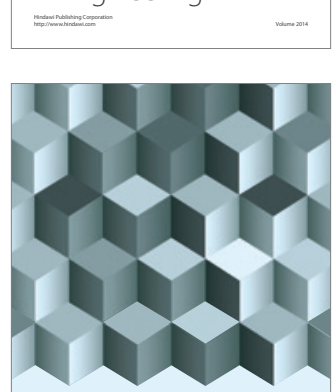

Journal of

Function Spaces
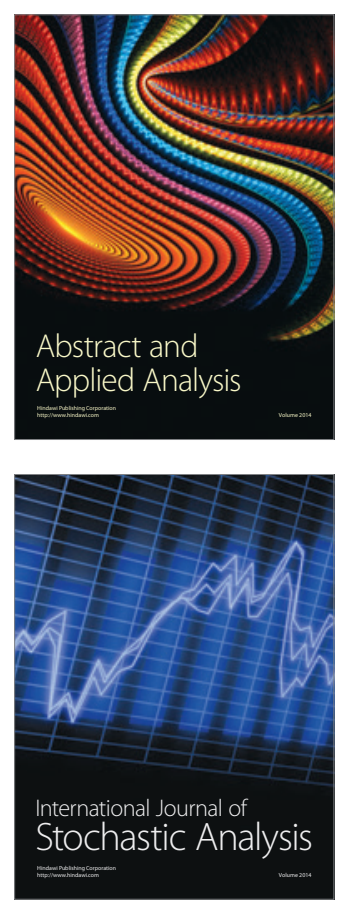

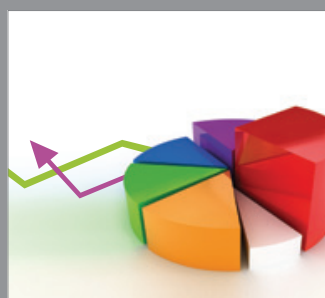

ournal of

Probability and Statistics

Promensencen
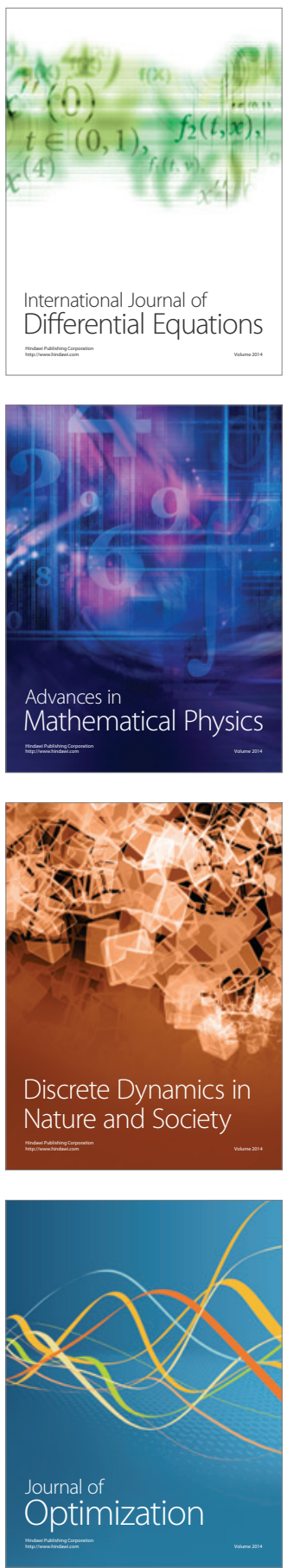